\title{
Delegation im Krankenhaus Entlastung durch Arztassistenz
}

Das Thema Delegation erfährt in jüngster Zeit neue Brisanz, da zugleich Möglichkeiten der Substitution diskutiert werden, die mit einer Neuordnung der Verantwortungsbereiche zwischen Medizin und Pflege verbunden ist. Was möglich ist, zeigt ein Projekt des Knappschaftskrankenhauses Bottrop. Es stützt sich auf ein trägerbezogenes Thesenpapier der Knappschaft Bahn See zur differenzierten fachlichen Abgrenzung von ärztlichen und pflegerischen Aufgaben, das von leitenden Ärzten und Pflegedirektoren aus den Trägereinrichtungen sowie unter Beteiligung des Deutschen Krankenhausinstituts erarbeitet worden ist. Pflegewissenschaftlerin Sabine Cittrich, Prozess- und Projektmanagerin in Bottrop, hat die Projektphasen begleitet.

\begin{abstract}
? Warum befasst sich das Knappschaftskrankenhaus Bottrop mit Delegation? Sabine Cittrich: Das Thesenpapier Knappschaft Bahn See hat die Betriebsleitung Sabine Cittrich der Klinik überzeugt.

Wir haben eine Grundlage erhalten, uns intensiv mit den Aufgabenbereichen der Medizin und Pflege und mit Delegationsmöglichkeiten zu befassen. Angesichts der Personalengpässe im ärztlichen und zunehmend auch im pflegerischen Bereich wird es immer wichtiger, die $\mathrm{Zu}$ friedenheit in den Berufsgruppen zu erhöhen und beispielsweise den Arztdienst von Verwaltungsaufgaben zu entlasten.
\end{abstract}

\section{? Wann und womit begann das Projekt?}

Cittrich: Das Projekt startete im Herbst 2010 mit einem Kickoff-Meeting für die Mitarbeiter der Klinik für Innere Medizin, in der das Projekt umgesetzt werden sollte. Bei dem Meeting haben der Geschäftsführer, Herr Tatka, und Herr König vom Deutschen Krankenhausinstitut die Projektziele und die beiden Projektarbeitsgruppen, je eine für Pflege und Medizin, vorgestellt. Im Vorfeld führte Herr König Gespräche mit Herrn Dr. Trenn, Chefarzt der Klinik, und mit Frau Degen, Pflegedirektorin, um die erforderliche Zustimmung einzuholen und die Inhalte des Aufgabenkatalogs abzuklären, die dann in den Gruppen zur Diskussion gestellt werden sollten. Bereits vor dem offiziellen Projektstart suchte Herr König das Gespräch mit dem Personalrat, um diesen frühzeitig einzubinden.

\section{? Wie sind die Projektgruppen} besetzt?

Cittrich: In der Projektgruppe Pflege waren 5 Pflegekräfte aus 3 Stationen und die zuständige Abteilungsleitung vertreten, in der anderen Gruppe 3 Ärzte. Die Arztgruppe ist kleiner, da die Ärzte stationsübergreifende Dienste haben. Der Personalrat, der das Projekt begleitende Vertreter des Trägers, Herr Erwig, Herr König als externer Projektleiter und ich als interne Projektleiterin haben die Projektsitzungen begleitet.

\section{Liegen Ergebnisse vor?}

Cittrich: Ergebnisse lagen bereits im Dezember 2010 vor. Die Projektgruppen haben intensiv gearbeitet und sich mehrmals über einen vierstündigen Zeitraum getroffen. Ziel war es, die vorgeschlagenen delegationsfähigen Leistungen auf den Prüfstein zu stellen und Konsens in den Fachgruppen zu erzielen. Im Großen und Ganzen haben sich die Vorschläge

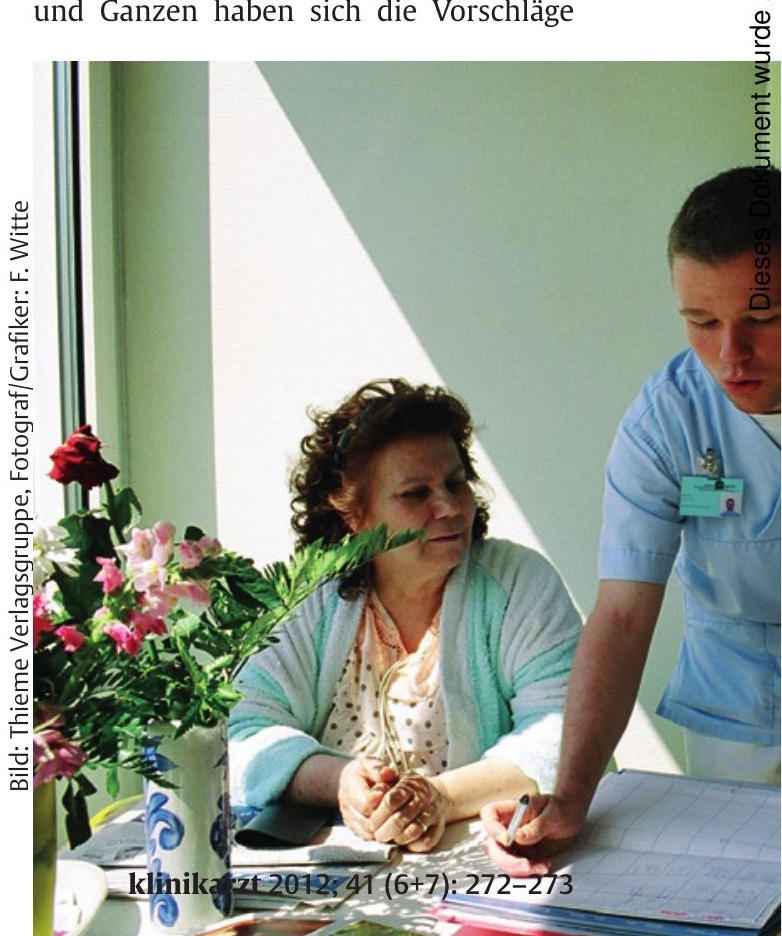


des Thesenpapiers bestätigt, ebenso wie die zu Beginn eingebrachten Ergänzungen von Herrn Dr. Trenn. Zum Teil sind delegierbare Tätigkeiten wie Blutabnahmen schon vorher von Assistenzkräften ausgeführt worden. Das Projekt hat dieses besser sichtbar gemacht.

\section{? Gab es Unterschiede in der} Arbeitsweise zwischen der Pflegegruppe und der Ärztegruppe?

Cittrich: Ja, die Ärztegruppe war sich ihrer Stellung im Wertschöpfungsprozess wesentlich mehr bewusst und konnte schneller delegationsfähige Aufgaben benennen. In der Pflege war es notwendig, sich zunächst grundsätzlich über den Wert der pflegerischen Arbeit zu verständigen. Wir haben die verschiedenen Bestandteile der pflegerischen Tätigkeit gesammelt, um uns einen besseren Überblick zu verschaffen. Anhand dieser Tätigkeiten wurden die Kategorien soziale, fachliche, personale und methodische Kompetenzen gebildet. Auch hier hat sich gezeigt, dass bereits viele Aufgaben an nachgeordnete Kräfte übertragen werden. Die originäre Aufgabe der examinierten Pflegekräfte besteht darin, den Patienten ganzheitlich wahrzunehmen und zu entscheiden, bei welchem Patienten welche pflegerischen Aufgaben delegiert werden dürfen und bei welchen dieses nicht verantwortbar wäre.

\section{? Zu welchen Konsequenzen haben} die Arbeitsergebnisse geführt?

Cittrich: Herr König hat einen Vorschlag erarbeitet, wonach Tätigkeiten von dem ärztlichen Bereich auf die Pflege übertragen werden sollen. Dieses sollte auch im

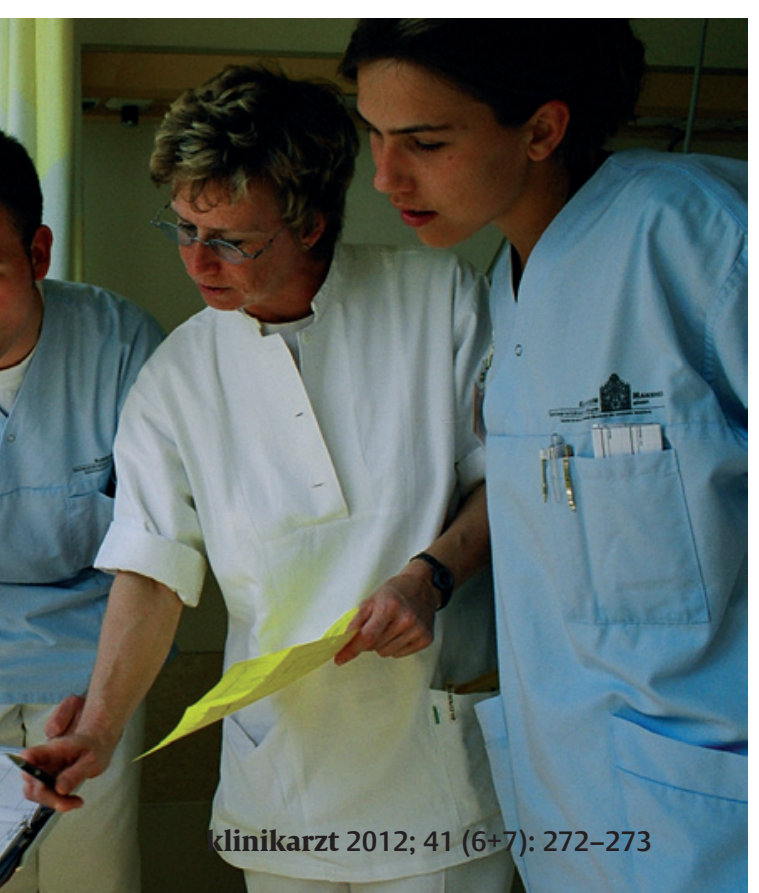

Stellenplan verankert werden, d.h., der ärztliche Dienst verliert eine Stelle, dafür erhält die Pflege 2 Stellen, die sie wiederum für examinierte Pflegekräfte oder nachgeordnetes Personal nutzen kann. Die Zuständigkeit für die delegierbaren Aufgaben sollte der Pflege obliegen, wobei die Verantwortung beim Arztdienst bleiben würde. Wesentlich ist, dass nicht einzelne Tätigkeiten isoliert betrachtet werden, sondern dass Aufgabenbereiche dauerhaft, 24 Stunden täglich, an 7 Tagen in der Woche, neu geregelt werden. Die Regelungen dürften nicht zurückgenommen werden, wenn wieder mehr Ärzte verfügbar sind.

\section{? Wie haben die Ärzte und Pflege-} kräfte den Vorschlag aufgenommen? Cittrich: Die Arbeit mit Delegationspotenzialen wurde sehr ernst genommen. Der Vorschlag von Herrn König fand jedoch keine Zustimmung. Die Ärzte kritisierten, dass Stellenanteile des Arztdienstes unwiederbringlich in den Pflegedienst übergehen würden. Seitens der Pflege bestand Sorge, dauerhaft zusätzliche Aufgaben und Verantwortung zu erhalten, hierfür aber nicht nachhaltig ausgestattet zu werden.

\section{? Was folgte dann?}

Cittrich: Stattdessen hat sich ein anderes Modell herauskristallisiert. In der Medizinischen Klinik wurden 2 Arztassistenten eingestellt, die direkt dem Arztdienst zugeordnet sind. Ihre Aufgaben sind genau definiert. Die Assistenten nehmen zum Beispiel Blut ab. Sie legen Venenkanülen, dokumentieren die Arzneimittelanamnese, begleiten Visiten, schreiben Bescheinigungen, füllen Reha-Anträge aus, führen Gespräche mit Hausärzten und Angehörigen und bereiten Patientenaufklärungen vor, sodass sich der Arzt im Patientengespräch noch gezielter auf spezielle Nachfragen und Besonderheiten konzentrieren kann.
Hat das Projekt zu weiterem Input für die Klinik geführt?

Cittrich: Das Modell der Arztassistenz bewährt sich gut. Es ist ein neues Berufsbild entstanden. 2 weitere Kliniken haben ebenfalls Arztassistentinnen eingestellt, darunter 2 Arzthelferinnen. Zur Entlastung des Pflege- und Arztdienstes ist eine Bettenmanagerin neu eingestellt worden, da bislang viel Zeit für die Suche nach freien Betten verloren ging. Ferner haben wir die Arbeitszeiten des Reinigungsdienstes ausgeweitet, damit nicht Pflegekräfte Betten und Schränke reinigen müssen, wenn Patienten erst nachmittags oder am Wochenende entlassen werden. Außerdem ist ein Qualitätszirkel entstanden, der sich insbesondere mit den Schnittstellen und dem beruflichen Selbstverständnis der Pflege befasst.

\section{Worauf führen Sie den Erfolg des Projektes zurück?}

Cittrich: Herr Dr. Trenn als Chefarzt und Frau Degen als Pflegedirektorin haben das Projekt von Anfang an unterstützt. Insbesondere den Assistenzärzten wurde die Angst genommen, ihnen würde etwas weggenommen. Wenn Ärzte und Pflegekräfte nicht dahinter stehen, ist die Gefahr groß, dass Delegationsprojekte scheitern.

\section{Ihr Ausblick?}

Cittrich: Die Resonanz ist bei allen Beteiligten positiv. Die Berufszufriedenheit der Ärzte steigt, und die Pflege hat einen großen Schritt in der Auseinandersetzung mit dem eigenen Wert und ihren Aufgaben gegenüber den Patienten gemacht. Das Projekt zeigt Entwicklungspotenziale auf. Eine der nächsten Aufgaben ist es, das Entlassungsmanagement stringenter zu fassen. Dies könnte durch Fallmanager geschehen.

Das Interview führte Dr. Adelheid Weßling, Düsseldorf

\section{Abonnementspreise 2013}

Der Verlag muss leider wegen der gestiegenen Herstellungs- und Vertriebskosten die Bezugspreise für den Jahrgang 2013 von klinikarzt moderat erhöhen.

Ab dem Januar 2013 berechnen wir für das Einzelheft 18,00€ und für das Jahresabonnement $124,00 €$. Arzte in Weiterbildung erhalten ein ermäßigtes Jahresabonnement für 84,00 $€$ und Medizinstudenten für 60,00 $€$ gegen Nachweis und Bankeinzug (alle Preise inkl. Mehrwertsteuer).

Im Ausland entstehen zusätzliche Versandkosten (cash with order) in Höhe von 41,80 $€$ innerhalb Europas und 76,90 € beim Versand nach Übersee/Airlift. 